\title{
Uso de autoevaluación docente como herramienta innovadora para el mejoramiento de las asignaturas universitarias*
}

Using faculty self-evaluation as an innovative tool to improve university courses

\author{
Katherine Ingrid Rico-Reintsch \\ PhD. (c) en Pensamiento Complejo, Multiversidad Mundo Real Edgar Morín, \\ Ciudad de México-México, kricoreintsch@gmail.com
}

\begin{abstract}
Cómo citar / How to cite
Rico-Reintsch, K. I. (2019). Uso de autoevaluación docente como herramienta innovadora para el mejoramiento de las asignaturas universitarias. Revista CEA, 5(10), 69-81. https://doi.org/10.22430/24223182.1445
\end{abstract}

Recibido: 9 de enero de 2019

Aceptado: 1 de abril de 2019

\section{Resumen}

Este articulo analiza los cambios realizados en el proceso enseñanza y aprendizaje durante 14 semestres (2012-2018), de la asignatura Diseño publicitario I, de la carrera de Publicidad y Marketing, de la Universidad Franz Tamayo sede Cochabamba. Para esto, se usó como instrumento de análisis la autoevaluación docente. Los datos se obtuvieron de tablas de observación que examinaron las estrategias didácticas usadas en cada curso; los resultados presentan que existió curación de contenidos acordes al contexto global multidimensional y complejo, investigación y co-creación de evaluaciones con los estudiantes, uso de tecnología como medio de práctica educativa y generación de nuevos espacios de aprendizaje físicos y virtuales. Adicionalmente, se realizó un sondeo de opinión a 30 docentes universitarios, que señaló que el 17 \% utiliza a la autoevaluación como referente de su práctica docente. Finalmente, se concluye que la autoevaluación docente puede ser usada como una herramienta de aprendizaje pertinente e innovadora para el docente y el estudiante.

Palabras clave: innovación educativa, herramientas de aprendizaje, prácticas educativas.

\begin{abstract}
This article analyzes the changes made to the teaching and learning process of the course Advertisement Design 101 in the Advertisement and Marketing program at Franz Tamayo University in Cochabamba, Bolivia, over a period of 14 semesters (2012-2018). Faculty self-evaluation was used for that purpose. The data were obtained from educational standards that describe the pedagogical strategies adopted in each course. The results show that their content curation is in line with their multidimensional and complex global context, research and co-creation of evaluations with students,
\end{abstract}

\footnotetext{
* Este artículo se deriva del proyecto titulado «Autoevaluación docente: una herramienta de aprendizaje pertinente e innovador» y ha sido financiado con recursos propios.
} 
the use of technology as a means of educational practice, and the generation of new physical and virtual learning spaces. Additionally, 30 faculty members participated in a survey, and $17 \%$ of them indicated they used self-evaluations as a reference point for their teaching practice. In conclusion, faculty self-assessment can be a relevant and innovative learning tool for professors and students.

Keywords: Educational innovation, learning tools, educational practices.

\section{INTRODUCCIÓN}

La autoevaluación es uno de los instrumentos que utilizan las Instituciones de Educación Superior (IES) para evaluar el desempeño profesional docente; también es una herramienta que permite incorporar una perspectiva propia del docente en la que da a conocer la forma como se valora y evalúa su práctica pedagógica.

Calatayud Salom (2008) apuesta por la autoevaluación como estrategia para desarrollar procesos de calidad de la práctica docente, que permitiría categorizar a los docentes dentro de una escala que va desde un simple transmisor de información, hasta llegar al nivel más alto como un valorador de la metacognición de su propio saber y de su práctica profesional. Asimismo, señala que es a partir de 1980 que comienza un creciente interés de la evaluación del profesorado, en una especie de continuo estado evaluador, afirmando que la evaluación de la práctica docente es un indicador de calidad del sistema educativo y una garantía de la calidad de la docencia.

Por otra parte, Calatayud Salom (2004) menciona que la autoevaluación del docente es una auténtica aventura, un apasionante viaje plagado de dificultades, contradicciones, pero también de posibilidades y satisfacciones, permiten reconducir y vislumbrar cuáles son los puntos fuertes y débiles de cada uno. Según la autora, el conocer la práctica docente ayuda a mejorar y a innovar en el trabajo diario con los estudiantes.

Bozu y Canto (2009) afirman que los docentes deben estar preparados para seleccionar, actualizar y utilizar el conocimiento en un contexto específico, ser capaces de aprender en diferentes contextos y modalidades a lo largo de toda la vida, para que puedan entender el potencial de lo que van aprendiendo y así adaptar el conocimiento a situaciones nuevas en sus aulas. Lo que no se autoevalúa se devalúa y ello no permite una innovación en la gestión de aula y en la creación de innovadoras, pertinentes y complejas situaciones en el proceso enseñanza y aprendizaje y sus diferentes componentes: docente, estudiante, currículo, aula, tecnología y contexto económico, político, sociológico, psicológico, afectivo, etc.

La sociedad en la actualidad está generando, desarrollando y difundiendo el conocimiento de forma exponencial, de forma que este saber se construye en la información adquirida a través de la experiencia o mediante el aprendizaje; por tanto, la labor del docente se convierte en ser un facilitador para localizar, gestionar y procesar dicha información como un vehículo y no como un conductor (Tobón, 2017).

Day (2006) afirma que la enseñanza apasionada tiene una función emancipadora que consiste en influir en la capacidad de los estudiantes ayudándoles a elevar su mirada más allá de lo inmediato, lo 
mismo que para aprender más sobre sí mismos; este compromiso firme y continuado con la enseñanza, así como con la satisfacción en el trabajo de docencia, evitará el estancamiento profesional.

Partiendo de la frase célebre del profesor y escritor estadounidense William Arthur Ward (1921 1994), «El profesor mediocre dice. El buen profesor explica. El profesor superior demuestra. El gran profesor inspira», se puede afirmar que el docente complejo se autoevalúa, entendiendo que existe complejidad cuando no se pueden separar todos los componentes diferentes que constituyen un todo en el proceso enseñanza y aprendizaje.

La Real Academia Española (2001) define 'docente' como «aquel que enseña», pero todos los que han sido tocados con la pasión y entrega en aula, por alguno de ellos, reconocen que hacen mucho más que «enseñar». La diversidad de docentes universitarios presenta a personas serias o sarcásticas, estrictas o más comprensivas; desentendidas o responsables; ausentes o siempre presentes; temerarias, tecnológicas, pasivas y activas, etc.; pero todas estas personalidades generan un impacto común: que dejan huella (positiva o negativa) en la vida del estudiante.

\section{MARCO TEÓRICO}

El desempeño o evaluación docente es reconocida en los últimos años como uno de los mecanismos determinantes para optimizar la calidad del trabajo de los docentes y, con ello, mejorar la calidad de la educación de los estudiantes que viene midiéndose desde que se acuñó el término calidad de educación. Para Cardona (2002), la calidad es el ideal de perfección que se persigue en las diferentes acciones educativas, es esa especie de tensión utópica que mueve y motiva, a la mejora permanente de todo cuanto se hace en el ámbito de lo educativo.

Martínez, Yániz-Álvarez y Villardón (2018) señalan la denominada «generación de la evaluación para la calidad», que ha promovido el desarrollo de una cultura de evaluación centrada en la mejora de la educación, donde el docente es la pieza clave en la mejora de la calidad educativa. Asimismo, afirman que poner en práctica propuestas de autoevaluación, además de resultar enriquecedor y respetuoso con la autonomía profesional docente, permitirá al propio profesorado autodirigirse hacia su desarrollo profesional.

El docente es un profesional de la educación obligado a reconstruirse (Montero y Gewerc, 2010) en mundo complejo. La complejidad implica una forma de dialogar con la realidad, más que tratar de abarcarla en su totalidad, afirma Yus (1998), y es por ello que estos procesos de enseñanza y aprendizaje deben llevar la realidad a las aulas, contextualizando el conocimiento.

Mas (2011) al hablar sobre el docente y sus competencias pedagógicas menciona entre las nuevas funciones del docente a la evaluación del proceso enseñanza y aprendizaje, y a la investigación, como un elemento de innovación en el mismo.

Por otra parte, dentro de lo que es la evaluación docente, Calatayud Salom (2018) presenta a la autoevaluación docente como aquella que facilita y beneficia el desarrollo y el crecimiento tanto personal como profesional, y que es una estrategia de mejora de la práctica docente. Asimismo, 
señala Calatayud Salom (2004) que por ello se trata de incidir en los principales interrogantes que el docente se puede plantear a la hora de querer iniciar procesos de autoevaluación, con el claro objetivo de proporcionar supuestos y herramientas que faciliten su viabilidad en el aula.

Tobón (2017) afirma que la evaluación socioformativa, nacida en Latinoamérica, es un proceso de diagnóstico, retroalimentación y apoyo continuo a las personas, para que aprendan a resolver problemas del contexto a través de la evaluación, co-evaluación y autoevaluación; es así como el modelo educativo por competencias instituye la autoevaluación del docente y del estudiante como una herramienta de reflexión, análisis y búsqueda de una construcción conjunta del aprendizaje entre ellos.

Resumiendo, la autoevaluación del docente resultará de un proceso reflexivo sobre su accionar en el proceso enseñanza y aprendizaje; sobre las competencias a ser alcanzadas por los estudiantes en un plan de formación formulado y el camino para alcanzarlas a través de la clase, en busca de un aprendizaje significativo y contextualizado.

Chacón (2014), respecto a los elementos que componen un proceso enseñanza aprendizaje y gestión educativa indica que es ante todo un sistema de saberes o competencias para la acción, un sistema de prácticas. Es decir, para que las organizaciones realmente puedan optimizar los frutos de su gestión es necesario pasar de una estructura de conocimiento simplista a una estructura de conocimiento compleja, donde todos los integrantes de la organización aporten su porción de conocimiento, lo que sería la verdadera civilización de las ideas.

El autor concluye que se debe hacer un tránsito de paradigma hacia la integración, la diversificación, la innovación y la creación de estrategias, que permitan asumir los cambios externos a la institución educativa, como al desarrollo temático y tecnológico de las propuestas de carrera para el siglo XXI, por ello se considera necesario penetrar el pensamiento complejo y la transdisciplinariedad dentro de las organizaciones y los diversos subsistemas del sistema educativo.

Lo mencionado por Chacón (2014) no explicita al docente como actor principal, pero lo nombra como integrante de la institución educativa; como uno de los móviles en busca de un pensamiento que integre los saberes con el contexto y las necesidades de la sociedad, en busca o como creador de estrategias que puedan desarrollar competencias; la autoevaluación docente buscará promover el desarrollo profesional y asegurar el cumplimiento de estándares institucionales para un desempeño de calidad y así mejorar los aprendizajes de los estudiantes.

Blázquez Andújar, et al (2007) plantean que la autoevaluación de la práctica docente es un proceso en el que los docentes realizan opiniones valorativas sobre la adecuación y efectividad de su propio conocimiento y actuación, con el fin de mejorar su intervención docente. Asimismo, señalan que la autoevaluación se centra en la práctica personal, en las propias experiencias sobre lo que significa ser docente y la propia necesidad de dar un sentido y mejorar esas prácticas del día a día en el aula. Concluyen que es el docente que está en el centro del proceso autoevaluador y se responsabiliza de examinar y mejorar su propia práctica docente.

Díaz-Barriga-Arceo \& Hernández Rojas (2007) señalan que es necesario contextualizar el aprendizaje acorde a la realidad social, ofreciendo estrategias prácticas para materializar tal propuesta; por su 
parte, Morín (2001) introduce la transdisciplinariedad y el pensamiento complejo, y plantea que el aprendizaje pertinente debe ser construido en los salones universitarios y no solo debe buscar ser contextualizado, significativo y continuo, sino también debe tomar en cuenta que todas y cada una de las personas son totalmente distintas, por tanto, el tipo de aprendizaje será contemplado por cada persona de forma individual y eso deberá ser tomado en cuenta por el docente a la hora de diseñar la construcción del proceso enseñanza y aprendizaje en aula.

Morín (2001) señala que para que un conocimiento sea pertinente debe evidenciar:

El contexto: ubicar las informaciones y los elementos en su contexto para que adquieran sentido.

Lo global: Es más que el contexto, es el conjunto que contiene partes diversas ligadas de manera inter-retroactiva u organizacional.

Lo multidimensional: La sociedad comporta dimensiones históricas, económicas, sociológicas, religiosas... El conocimiento pertinente debe reconocer esta multidimensionalidad e insertar allí sus informaciones: se podría no solamente aislar una parte del todo sino las partes unas de otras.

Lo complejo: Complexus significa lo que está tejido junto; en efecto, hay complejidad cuando son inseparables los elementos diferentes que constituyen un todo (como el económico, el político, el sociológico, el sicológico, el afectivo, el mitológico) y que existe un tejido interdependiente, interactivo e interretroactivo entre el objeto de conocimiento y su contexto, las partes y el todo, el todo y las partes, las partes entre ellas. (Morín, 2001, p.15).

Morín (2001) plantea que será necesario reformular la enseñanza y por ende la evaluación desde los siguientes principios:

1. La intersubjetividad, entendiendo que el conocimiento no es inamovible y objetivo, sino subjetivo e interrelacional.

2. La interactividad, a partir de la cual sabremos que cualquier acción, una vez hecha, entra en un juego de interacciones y retroacciones en el medio donde se efectúa y puede desviarse de sus objetivos, incluso puede llevarla a un resultado contrario al previsto.

Para Morín (2001) el conocimiento es una aventura incierta que conlleva en sí misma y permanentemente el riesgo de ilusión y de error, por lo que es función del docente verificarlo a través de la planificación, diseño y creación de actividades didácticas dentro del proceso enseñanza y aprendizaje para construirlo. Es el docente quien debe percibir las fuentes de error, facilitando para sí mismo las oportunidades de crecer con cada asignatura.

Por su lado, Freire (2004) indica que: «el progreso científico y tecnológico que no responde fundamentalmente a los intereses humanos, a las necesidades de nuestra existencia, pierde, para mí, su significación». Por tanto, deberá buscarse un proceso enseñanza y aprendizaje que busque suplir esas necesidades en busca de innovación, tanto en el proceso enseñanza aprendizaje como del conocimiento. 
Sintetizando a todos los autores mencionados, se pude señalar que la autoevaluación docente debe ser una notable práctica educativa que debiera ser originada en la co-creación de saberes con los estudiantes; en el análisis del registro generado de, y por la experiencia en aula, y lo más importante: por la búsqueda de una construcción compleja de saberes significativos y contextuales dentro de la universidad para responder las necesidades de la sociedad.

\section{METOdOLOGÍA}

El objetivo de la investigación fue el conocer si la autoevaluación docente es una herramienta pertinente e innovadora a la hora de planificar un nuevo plan de clases para la asignatura en la siguiente gestión.

El enfoque de la investigación fue mixta cualitativa con el uso de tablas de observación, y cuantitativa por el sondeo de opinión. El tipo de estudio fue descriptivo. Como se trata de un estudio de caso, la población fue al mismo tiempo la muestra con la que se trabajó: los estudiantes inscritos en la asignatura de «Diseño Publicitario |», del primer semestre de la carrera de Publicidad y Marketing, durante los 14 semestres.

El método que se utilizó para evaluar los criterios de autoevaluación fue analítico, a través del cumplimiento de variables planteadas en tablas de observación. Se analizó las clases por semestre para luego reflexionar sobre las estrategias didácticas que fueron apropiadas a las características de los estudiantes, y así identificar los aspectos efectivos del proceso enseñanza y aprendizaje, también se consideró los contenidos de la asignatura que necesitaban ser contextualizados.

Asimismo, se analizó el diseño de las evaluaciones (para la asignatura elaboración de materiales) y su relación directa con los resultados de aprendizaje, verificando las consignas, orientación y las herramientas de evaluación que identifican con precisión las competencias esperadas. Se verificó las evaluaciones para entender cómo influyeron las decisiones didácticas en los aspectos, tanto logrados como los no logrados en los estudiantes, además se analizó la entrega oportuna de retroalimentación específica y útil para que los estudiantes mejoren sus aprendizajes.

Para elaborar las variables de autoevaluación se usaron parámetros propios y los mencionados por Morín (2001):

a. Conocimiento pertinente. Es aquel capaz de situar cualquier información en su contexto y, si puede ser, en la globalidad en la que se inscribe. El conocimiento nada más es conocimiento en tanto que organización, relación y contextualización de informaciones. Las informaciones no son más que parcelas de saber dispersas. Por restringida que sea la materia, el especialista ni siquiera puede llegar a tener conocimiento de toda la información dedicada a su área. Cada vez más, la proliferación gigantesca de conocimientos se escapa al control humano.

b. Aprensión de la realidad. El conocimiento es organización, relación y contextualización de informaciones.

c. Valoración del error. Estos dos elementos: subjetividad de la evaluación y toma de decisiones que modifiquen el proceso de aprendizaje. 
d. Auto-provocación del éxito. La finalidad última de la evaluación será la de guía en el camino complejo que se emprende.

Entre los parámetros propios se tomaron en cuenta:

1. Papel del docente como orientador de aprendizaje, curador de contenidos y promotor de investigación.

2. Rol del estudiante como co-creador de evaluaciones, permitiendo que tengan voz los estudiantes, respecto a co-construir un nuevo saber.

3. Usar a la tecnología con medio de práctica educativa.

4. Promover el análisis y la interpretación de resultados en los estudiantes como parte de su aprendizaje pertinente.

5. Generación de espacios de aprendizaje: ambientes físicos y virtuales.

Se realizó un sondeo de opinión (técnica cuantitativa) para conocer si otros docentes realizaban una autoevaluación propia o institucional y así comprender la importancia y uso que le dan los docentes a la misma en su práctica profesional.

\section{RESULTADOS}

Los resultados del análisis de los cambios realizados en el proceso enseñanza y aprendizaje, ejecutados durante los 14 semestres (2012-2018) en que se dictó la asignatura «Diseño Publicitario |» de la carrera de Publicidad y Marketing, son presentados bajo la dirección teórica de Blázquez Andújar, et al (2007) y Morín (2001), verificando la teoría en la práctica a través de la autoevaluación docente.

La Tabla 1 presenta la síntesis de las tablas de observación cualitativas de los dos más grandes cambios realizados en los 14 semestres detallados por año en la parte académica, tecnológica y administrativa. Resume las principales acciones y logros del proceso enseñanza y aprendizaje en las distintas gestiones en las que se dictó la asignatura, resumiendo la re-estructuración académica (inclusión teórico práctica de materiales), tecnológica (uso de plataformas virtuales) y administrativas (modelo de la universidad e investigación) realizada a través de la autoevaluación docente.

La Tabla 1 aclara la progresión cada semestre, de la elaboración del principal resultado: un «Portafolio de diseñador junior», que presenta 10 materiales publicitarios y 10 de relaciones públicas innovadores, elaborados para una empresa local, en dos idiomas: español e inglés, con sus respectivas fichas técnicas y de proceso para que puedan ser reproducidas. Además, presenta la introducción de la gamificación y creación del premio Uf Design entregado semestralmente al estudiante (diseñador publicitario), como premio a su portafolio y los materiales presentados en él. 
Tabla 1. Reestructuración académica - tecnológica - administrativa asignatura Diseño Publicitario I Table 1. Academic-technological restructuring of the course Advertisement Design 1

\begin{tabular}{|c|c|}
\hline Semestre & Reestructuración académica \\
\hline 2012-1 & $\begin{array}{l}\text { Se mantuvo el contenido básico de la asignatura, se llevó la teoría a la práctica con la } \\
\text { elaboración de cinco materiales publicitarios. El principal resultado, la motivación de la } \\
\text { aplicación del conocimiento al elaborar materiales. }\end{array}$ \\
\hline $2012-2$ & $\begin{array}{l}\text { Se incrementó el número de materiales publicitarios elaborados para la empresa de cinco a } \\
\text { siete. El resultado fue el análisis de la pertinencia del uso de los materiales nuevos para la } \\
\text { empresa por parte de los estudiantes. }\end{array}$ \\
\hline 2013-1 & $\begin{array}{l}\text { En este semestre se introdujo tres materiales de relaciones públicas y se comenzó con la } \\
\text { elaboración de dos versiones de los materiales: uno tradicional (común) y uno innovador } \\
\text { (creativo que cause impacto en el público objetivo). Los resultados fueron mayor motivación } \\
\text { en los estudiantes por la creación de materiales innovadores y el cumplimiento de otros } \\
\text { objetivos como los que tienen los materiales de las relaciones públicas. }\end{array}$ \\
\hline $2013-2$ & $\begin{array}{l}\text { Se realizó como examen final de semestre un «Portafolio de Diseño», conteniendo } 12 \text { piezas } \\
\text { publicitarias y de relaciones públicas en versión tradicional e innovadora. El resultado fue que, } \\
\text { desde la creación del «Portafolio de Diseñador Junior», en cada semestre uno o dos } \\
\text { estudiantes por semestre consiguen un trabajo de diseño de materiales para una empresa. }\end{array}$ \\
\hline 2014-1 & $\begin{array}{l}\text { Se incluyen al Portafolio tres nuevos materiales acorde al contexto de las empresas con las } \\
\text { que los estudiantes están trabajando. Los resultados son la satisfacción de las empresas con } \\
\text { la creación de materiales que cubran sus objetivos publicitarios y de relaciones públicas. }\end{array}$ \\
\hline $2014-2$ & $\begin{array}{l}\text { Para trabajar interdisciplinariedad, se introdujo el idioma inglés con la elaboración de cinco } \\
\text { materiales escritos en inglés. El resultado es la valoración positiva de las empresas al elaborar } \\
\text { materiales bilingües. }\end{array}$ \\
\hline
\end{tabular}
Se incluyeron dos y tres materiales de relaciones públicas por cada gestión, haciendo un total 2015-1/2015-2 de 20 (10 piezas publicitarias y 10 de relaciones públicas) de los cuales siete estaban traducidos al inglés. El resultado mayor conocimiento práctico y de aplicación pertinente para los estudiantes y sus empresas.

Se trabajó en equipo con el docente que dictaba inglés, para que una parte de la evaluación

2016-1 de los estudiantes en su asignatura sea la escritura de los diez materiales de diseño publicitario. El resultado es favorable para los estudiantes al obtener dos notas para un mismo trabajo.

Se buscó el trabajo multidisciplinario docente, intercambiando evaluaciones, co-evaluando con la asignatura de «Análisis Económico», en el diseño de una carátula ejemplificativa para

el ensayo de una temática boliviana, la asignatura de «Análisis Económico» evaluaba el fondo y «Diseño Publicitario l» la forma del mismo trabajo. Como resultado primordial, se obtuvo la generación de trabajos complementarios y contextualizados al entorno y carrera para dos asignaturas.

Junto a los 20 materiales trabajados, se incluyó el elaborar fichas proceso para cada material, esta ficha presenta el boceto de creación de la pieza, el tiempo y los recursos que se utilizarán

2017-1 para producirlo, fue implementado para que los estudiantes aprendan a valorar el esfuerzo en tiempo y recursos utilizados. El resultado principal fue el que los estudiantes valoraron su tiempo y recursos, y así reconocieron mayor profesionalidad en la elaboración de sus materiales.

Se incluyó la producción de fichas técnicas para cada material, para que cualquier otro diseñador publicitario pueda reproducir el material usando como mapa la ficha técnica. El

2017-2 resultado fue que los estudiantes fueron precisos en colocar los elementos técnicos como tamaño, uso de color, tipo de materiales, etc., con los que elaboraron sus materiales para que pueda ser reproducido.

Por las características del entorno y el contar con dos paralelos de la asignatura, se introduce

2018-1 la gamificación con la creación de un premio de diseño junior, denominado UF DESIGN. La gamificación generó como resultados: el trabajo en equipo entre estudiantes, la interacción 
entre pares docentes invitados como jueces de la competencia y la creación de una propuesta innovadora de pieza publicitaria por parte de los estudiantes acorde a la empresa con la que se estaba trabajando para obtener el premio.

En este semestre se siguió con la gamificación personalizada, y para ganar la segunda versión del UF DESIGN los estudiantes deberían elaborar una propuesta de una pieza publicitaria y

2018-2 una de relaciones públicas. El resultado, buscar a través de la investigación el proponer el diseño de nuevas piezas publicitarias específicas para satisfacer las necesidades publicitarias y de relaciones públicas de una empresa.

\section{Cambios tecnológicos - administrativos}

Aplicación del modelo pedagógico por objetivos, uso de redes sociales - Facebook -, como herramienta de comunicación. El resultado, estudiantes que elaboran materiales contextualizados, apoyados por canales directos de comunicación con el instructor de los mismos.

$2012-2015$ Se implementó el uso del Facebook como plataforma educativa para poder tener una

2016 interacción más personalizada con los estudiantes. El resultado, mayor conocimiento del trabajo de los estudiantes y guía especifica.

La universidad implementó el modelo por competencias, y se usó la rede social Facebook como plataforma para aula invertida, a través de la creación de un grupo cerrado con solo los

2017-2018 miembros del curso, donde se presentaban los contenidos a ser trabajados en clases: vídeos, pdf, etc. El resultado, estudiantes más autónomos por tener guías específicas y ejemplificativas de cada material a ser trabajado disponibles en todo tiempo en la red.

A causa de la implementación de la gamificación, se les dio un nuevo uso a los grupos de Facebook como herramienta de co-valoración entre paralelos y grupos externos de

2018-1 estudiantes y de docentes para valorar los elementos de la competencia UF DESIGN. EI resultado, estudiantes de cursos paralelos conectados, docentes de la carrera conectados con estudiantes y evaluadores externos valorando diseños para ser utilizados por empresas locales.

Se implementó el usó de la plataforma gratuita Schoology donde se colocaron en línea todos los documentos teóricos y ejemplificativos a ser trabajados en la asignatura, asimismo la

2018-2 plataforma contiene todos los materiales realizados por cada estudiante en carpetas personalizadas. El resultado primordial, la creación de un portafolio digital de cada estudiante y la posibilidad de realizar evaluaciones en línea de parte del docente.

Fuente: elaboración propia.

Como conclusión de los resultados, se puede señalar que la Tabla 1 sintetiza la pertinencia (contexto) y la innovación de la temática (publicidad digital, curación y creación de contenidos e investigación) y las estrategias didácticas (analógicas y digitales) en el proceso enseñanza y aprendizaje bajo los parámetros ya citados por Morín (2001) en busca del aprendizaje pertinente, aprehensión de la realidad, valoración del error, interdisciplinariedad y auto-provocación al éxito, verificadas en la autoevaluación docente con el uso de las estrategias didácticas y actividades que se implementaron en aula, así como las reacciones, actitudes, interacciones y resultados que generaron los estudiantes como respuesta a las propuestas implementadas.

La síntesis de los resultados obtenidos es la siguiente:

- Papel del docente como orientador de aprendizaje, curador y creador de contenidos y promotor de investigación; encontrando nuevos autores y materiales, otros ejemplos y perspectivas para ser compartidas en clases a través de exposiciones.

- Rol del estudiante como co-creador de evaluaciones, permitiendo que tengan voz los estudiantes, respecto a co-construir un nuevo saber; a través del análisis de los materiales 
existentes en el contexto y su uso, utilizando la investigación que realizaron los estudiantes a la hora de plantear la introducción de nuevos materiales a ser evaluados.

- Usar a la tecnología con medio de práctica educativa; implementando el uso primero de la red social Facebook como plataforma educativa y posteriormente a Schoology como plataforma gratuita.

- Promover el análisis y la interpretación de datos en los estudiantes como parte de su aprendizaje pertinente; mediante la elaboración de piezas críticas dirigidas a públicos de un contexto global multidimensional y complejo en busca de la innovación de materiales competitivos y de alto impacto.

- Generación de espacios de aprendizaje: ambientes físicos y virtuales. Por medio de la visita de espacios físicos fuera de aula en busca de nuevas piezas publicitarias y de relaciones públicas y la búsqueda en la red de nuevas propuestas en el área.

El sondeo de opinión buscaba encontrar a aquellos docentes que realizaban autoevaluación, los resultados cuantitativos obtenidos al respecto se presentan en la Tabla 2, con la pregunta abierta: ¿Quiénes deberían evaluar la labor docente en el proceso enseñanza y aprendizaje? Se analizaron 30 respuestas de docentes universitarios de universidades públicas y privadas principalmente de Cochabamba, Bolivia. El 43 \% de los docentes señaló que la evaluación, principalmente, debe recaer en los estudiantes y la institución, y el 17 \% mencionó la autoevaluación sola o acompañada con otro evaluador; por ende, se puede concluir que la autoevaluación no es considerada por la mayoría de los docentes como un indicador para su práctica docente.

Tabla 2. Sondeo de opinión docentes universitarios

Table 2. Results of the survey of college professors

\begin{tabular}{lc}
\hline ¿Quiénes deben evaluar a los docentes en el proceso enseñanza y aprendizaje? \\
\hline Los estudiantes & 4 \\
\hline Los estudiantes y la institución & 13 \\
\hline Los estudiantes y autoevaluación & 1 \\
\hline Los estudiantes y pares docentes & 2 \\
\hline Los estudiantes, la institución y pares docentes & 3 \\
\hline Los estudiantes, pares docentes y un evaluador externo & 2 \\
\hline Los estudiantes, pares docentes y autoevaluación & 1 \\
\hline Pares docentes & 1 \\
\hline Pares docentes y autoevaluación & 2 \\
\hline Autoevaluación docente & 1
\end{tabular}

De los resultados del Tabla 2, se puede concluir que 13 de los docentes (43\%) señalan que los encargados de evaluar a los docentes deberían ser la institución y los estudiantes; 4 de los docentes (13 \%) señalan que solo estudiantes deben evaluarlos; y un docente $(0,03 \%)$ afirmó que la autoevaluación debe realizarla el mismo docente. 


\section{CONCLUSIONES}

El Marco para la calidad en la enseñanza reconoce la complejidad de los procesos de enseñanza y aprendizaje, y los variados contextos culturales en que estos ocurren; los docentes que se autoevalúan reconocen que los estudiantes tienen diferentes características, vienen de distintos contextos y tales condiciones y particularidades deben ser consideradas al momento de construir nuevos saberes con ellos en aula, por tanto, la autoevaluación se convertirá en una herramienta de aprendizaje pertinente porque analiza el contexto, y será innovadora debido a que buscará nuevas formas de construcción de saberes que se convertirán en reto para el docente y por ende al estudiante.

Fue la autoevaluación docente en la asignatura de «Diseño Publicitario |», la que demuestra que la participación del docente en el modelo por objetivos puede ser sustituida por herramientas de seguimiento (Facebook) y organización (Schoology) para comunicación e interacción entre docente y estudiante fuera de aula. La presencia física del docente en el modelo por competencias está para ser parte del equipo de enseñanza y aprendizaje: docente-estudiantes.

La autoevaluación docente significa construir y reconstruir; separar y concentrar; analizar y sintetizar el currículo contextualizarlo, gestionar el aula y al mismo tiempo presentar lo que se quiere realmente sea aprehendido generando un aprendizaje pertinente e innovador; por tanto, se concluye que cada docente podrá no solo utilizar la autoevaluación que le pueda dar su institución educativa, sino por su experiencia crear el propio método de autoevaluación que siga parámetros que midan sus competencias docentes en el siglo XXI, en busca de la innovación y la pertinencia globalizada y complejizada (Blázquez Andújar, et al, 2007).

Autoevaluarse, para un docente con parámetros complejos, hace que se construya o reconstruya con el estudiante un proceso de enseñanza y aprendizaje con una perspectiva compleja de ida y vuelta; donde se estructuran y reestructuran saberes, sentimientos, emociones, actitudes, expectativas y un conglomerado de diversos aspectos que tienen una finalidad: construir con el otro un saber.

Los procesos de evaluación que se realicen para los resultados de aprendizaje de la asignatura deben responder a la complejidad del aprendizaje y fomentar la auto-evaluación del estudiante y del docente, para ayudar a construir el conocimiento de la realidad que se desarrolla y evoluciona en su mente y en la del estudiante.

Plantear una docencia donde se le conceda mayor importancia al rendimiento académico, a la valoración estudiantil e institucional más que a la autoevaluación docente; crea asignaturas estancadas en el modelo pedagógico, o en currículo; generando repetidores de conocimiento y no estudiantes investigadores co-creadores de saberes teóricos, prácticos, emocionales y contextuales útiles para la profesión elegida y para la sociedad.

La auto-evaluación docente permitirá un aprendizaje pertinente e innovador, tanto para el estudiante como para el docente, en tanto sea construido por ambos, en pleno conocimiento del rol que tienen en esa construcción siempre en busca de la innovación, es decir, creatividad en acción. 
Se debe enfatizar que una herramienta como la autoevaluación debe estar dentro de una gestión educativa que involucre a la institución como parte del proceso enseñanza y aprendizaje; debe involucrar a los pares docentes en busca de una integral y compleja comprensión de los cambios del mundo estudiantil y de las constantes permutaciones técnicas y tecnológicas de las carreras universitarias para la sociedad. Debe incentivar el diálogo profesional en torno a las prácticas pedagógicas que ocurren como resultado de la evaluación docente. Por otra parte, esta autoevaluación no puede hacerse solo bajo parámetros teóricos o prácticos propios; debe estar acoplada a los principios, misión, visión y modelo educativo de la institución y del sistema educativo contextual al que pertenece.

Asimismo, comprender a la autoevaluación docente dentro del pensamiento complejo, es y será una herramienta clave, estratégica y articulada dentro una gestión educativa para enfrentar los retos de educación universitaria del siglo XXI.

Finalmente, la Tabla 2 cualitativamente analizada puede concluir que solo cinco de 30 docentes toman en la cuenta la autoevaluación a la hora de entender su práctica docente y, por tanto, es posible que mantengan por varias gestiones las mismas estrategias didácticas y formas de evaluación sin analizar la pertinencia y buscar la innovación temática, tecnológica y pedagógica.

\section{REFERENCIAS}

Blázquez Andújar, P. J., Cassé Tomás, J. L., Díaz Alcaraz, F., Escudero Díaz, J., Martinez López, A. M., Peña Gómez, F., et al. (2007). Modelo para autoevaluar la práctica docente (dirigido a maestros de infantil y primaria). Madrid, España: Wolters Kluwer.

Bozu, Z. y Canto, P. (2009). El profesorado universitario en la sociedad del conocimiento: competencias profesionales docentes. Revista de Formación e Innovación Educativa Universitaria, 2(2), 87-97. Recuperado de http://refiedu.webs.uvigo.es/Refiedu/Vol2 2/REFIEDU 2 2 2 4.pdf

Cardona, J. (2002). La evaluación y la calidad de los centros. En S. Castillo (Coord.). Compromisos de la evaluación educativa. Madrid, España: Pearson Educación.

Chacón, M. L. (2014). Gestión educativa del siglo XXI: bajo el paradigma emergente de la complejidad. Omnia, 20(2), 150-161. Recuperado de http://www.redalyc.org/articulo.oa?id=73735396006

Calatayud Salom, M. A. (2004). La autoevaluación de la práctica docente: una aventura plagada de dificultades y satisfacciones. Revista Ciencias de la Educación, (198-199), 151-170. Recuperado de https://core.ac.uk/download/pdf/41574770.pdf

Calatayud Salom, M. A. (2008). Establecer la cultura de la autoevaluación. Revista Padres y Maestros, 314, 30-34. Recuperado de https://revistas.comillas.edu/index.php/padresymaestros/article/view/1559 
Calatayud Salom, M. A. (2018). La autoevaluación. Una propuesta formativa e innovadora. Revista Iberoamericana de Educación, 76(2), 135-152. Recuperado de https://doi.org/10.35362/rie7623081

Day, C. (2006). Pasión por enseñar, la identidad personal y profesional del docente y sus valores. Madrid, España: Editorial Narcea.

Díaz-Barriga-Arceo, F. \& Hernández Rojas, G. (2007). Estrategias docentes para un aprendizaje significativo. México: Mc Graw Hill.

Freire, P. (2004). Pedagogía de la autonomía. Sao Paulo, Brasil: Paz e Terra.

Mas, O. (2011). El profesor universitario: sus competencias y su formación. Profesorado, Revista de currículum y formación del profesorado, 15(3), 195-211. Recuperado de http://www.redalyc.org/articulo.oa?id=56722230013

Martínez-Izaguirre, M., Yániz-Álvarez de Eulate, C., \& Villardón-Galleg, L. (2018). Autoevaluación y reflexión docente para la mejora de la competencia profesional del profesorado en la sociedad del conocimiento. RED. Revista de Educación a Distancia, 56. http://dx.doi.org/10.6018/red/56/10

Montero, L., y Gewerc, A. (2010). De la innovación deseada a la innovación posible. Escuelas alteradas por las TIC. Profesorado. Revista de Curriculum y Formación del Profesorado, 14(1), 303-318. Recuperado de http://hdl.handle.net/10481/7129

Morín, E. (2001). Los siete saberes necesarios para la educación del futuro. Bogotá, Colombia: UNESCO-Editorial Magisterio.

Real Academia Española. (2001). Diccionario de la lengua española (22. ${ }^{a}$ ed.). Madrid, España.

Tobón, S. (2017). Evaluación socioformativa. Estrategias e instrumentos. Mount Dora (USA): Kresearch.

Yus, R. (1998). Temas transversales: Hacia una nueva escuela (2a ed). Barcelona. España: Editorial Graó. 